\title{
Expression of p27Kip1, A Cell Cycle Repressor Protein with Dual Roles for Both Cancer Prevention and Promotion, Is Regulated Primarily at the Level of Unusual p27Kip1 mRNA-A Short Concept Proposal
}

\author{
Isao Eto ${ }^{1,2}$ \\ ${ }^{1}$ Department of Nutrition Sciences, University of Alabama at Birmingham, Birmingham, AL, USA \\ ${ }^{2}$ Webb Nutrition Sciences Building, University Boulevard, Birmingham, AL, USA \\ Email: etoi@uab.edu
}

How to cite this paper: Eto, I. (2018) Expression of p27Kip1, A Cell Cycle Repressor Protein with Dual Roles for Both Cancer Prevention and Promotion, Is Regulated Primarily at the Level of Unusual p27Kip1 mRNA-A Short Concept Proposal. American Journal of Molecular Biology, 8, 186-193.

https://doi.org/10.4236/ajmb.2018.83016

Received: June 25, 2018

Accepted: July 21, 2018

Published: July 24, 2018

Copyright $\odot 2018$ by author and Scientific Research Publishing Inc. This work is licensed under the Creative Commons Attribution International License (CC BY 4.0).

http://creativecommons.org/licenses/by/4.0/

\begin{abstract}
The p27Kip1 is a cell cycle repressor protein that regulates primarily the cell cycle transition from $\mathrm{G} 1$ to $\mathrm{S}$ phase and hence the DNA replication is in the $\mathrm{S}$ phase and cell division in the $\mathrm{M}$ phase. Expression of p27Kip1 protein has dual roles for both cancer prevention and promotion. For example, numerous nutritional and chemopreventive anti-cancer agents specifically increase the expression of p27Kip1 protein without directly affecting the expression of any other cell cycle regulatory proteins. On the other hand, pro-cancer agents (like glucose, insulin and other growth factors frequently seen in obesity and/or diabetes) specifically decrease the expression of p27Kip1 protein without directly affecting the expression of any other cell cycle regulatory proteins. Unlike expression of any other cell cycle regulatory proteins, expression of p27Kip1 protein is very unusual. The mRNA of p27Kip1 has a very long and unusual 5 -untranslated region (from -575 to -1 in human). It appears that the 5'-untranslated region of p27Kip1 mRNA forms two alternative secondary structures. One increases the expression of $\mathrm{p} 27 \mathrm{Kip} 1$ protein when anti-cancer agents are added and another decrease the expression of $\mathrm{p} 27 \mathrm{~K} 1 \mathrm{p} 1$ when pro-cancer agents are added. For this short concept proposal, Dr. Albert Einstein's "visualized thought experiments (German: Gedanken experiment)" were used as a fundamental tool for understanding how either anti- or pro-cancer agents bring the primary structure of the 5'-untranslated region of p27Kip1 mRNA into two alternative secondary structures, thereby either increasing or decreasing, respectively, the translation initiation of p27Kip1 protein.
\end{abstract}




\section{Keywords}

p27Kip1, Cell Cycle Repressor Protein, Cancer Prevention, Anti-Cancer Agents, Cancer Promotion, Pro-Cancer Agents, p27Kip1 mRNA, 5-Prime-Untranslated Region, Translation Initiation, 5-Prime Cap, Upstream Open Reading Frame, Internal Ribosome Entry Site

\section{Very Long and Unusual Primary Structure of 5'-Untranslated Region of p27Kip1 mRNA}

\subsection{Function of p27Kip1 Protein in the Cell Cycle (Figure 1)}

p27Kip1 is a cell cycle repressor protein with molecular mass of approximately $27 \mathrm{kDa}$. During the cell cycle, p27Kip1 primarily regulates the G1-to-S phase transition. When the expression of p27Kip1 protein is up-regulated, G1-to-S phase transition of the cell cycle, DNA replication in the $S$ phase and cell division in the $M$ phase are all repressed. This is how numerous nutritional and chemopreventive agents decrease the risk of developing cancer [1] [2] [3] [4] (for further references about p27Kip1 in general, see the Introduction sections of the reference Nos. [1] [2] [3] [4] [5]).

On the other hand, when the expression of p27Kip1 protein is down-regulated, G1-to-S phase transition of the cell cycle, DNA replication in the S phase and cell division in the $\mathrm{M}$ phase are all un-repressed. This is how several pro-cancer agents, including glucose, insulin, other growth factors and certain amino acids frequently seen in obesity and/or diabetes increase the risk of developing cancer [1] [2] [3] [4] [5].

Anti- or pro-cancer agents specifically either increase or decrease, respectively, the risk of developing cancer without directly affecting the expression of any

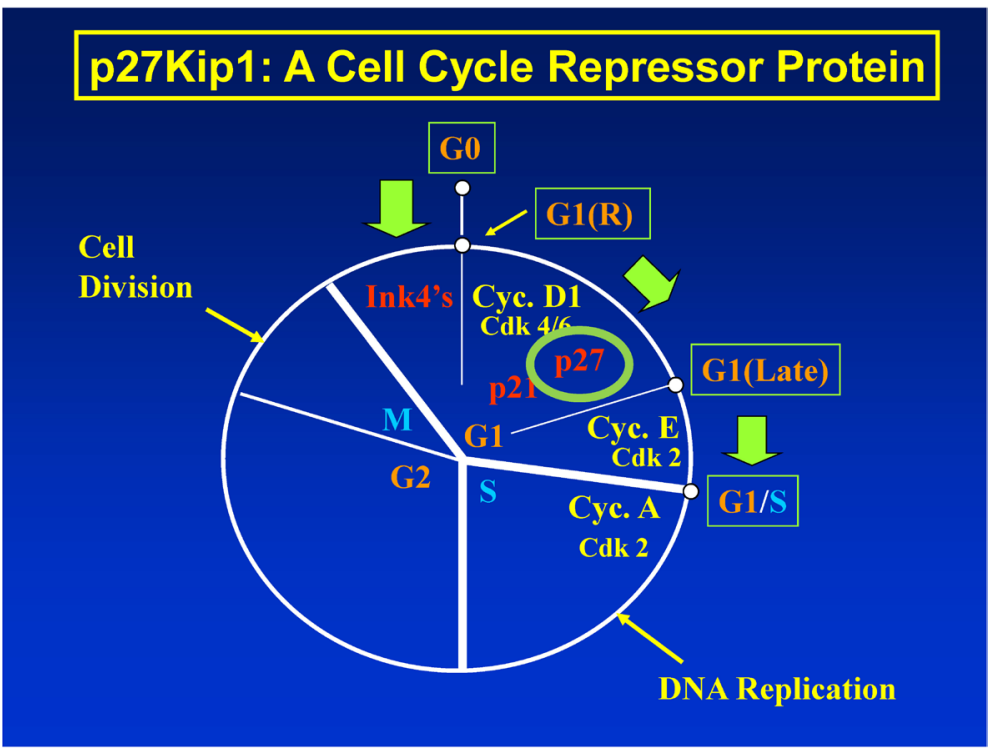

Figure 1. Function of p27Kip1 protein in the cell cycle. 
other cell cycle regulatory proteins including p21Cip1/Waf1 [1].

\subsection{Primary Structure of 5' -Untranslated Region of p27Kip1 mRNA (Figure 2)}

Unlike expression of any other cells cycle regulatory proteins, the expression of p27Kip1 protein is very unusual. The mRNA of p27Kip1 has a very long 5 '-untranslated region (5'-UTR) (from -575 to -1 in human) [2]. This very long 5'-UTR of p27Kip1 mRNA is the key to gain understanding of the basic molecular mechanisms of how either 1) anti-cancer agents decrease the risk of developing cancer or 2) pro-cancer agents increase the risk of developing cancer.

The primary structure of the 5'-UTR of human p27Kip1 mRNA is shown in Figure 2. It contains three distinct elements [2]:

a) The first element is the five-prime cap (5'-cap) located at -575 . The $5^{\prime}$-cap is 7 -methylguanylated. So, it is usually abbreviated as 7-methylguanylated 5 '-cap (m7G-5'-cap). One of the general functions of $\mathrm{m} 7 \mathrm{G}-5^{\prime}$-cap is to protect mRNA from degradation.

b) The second element is the upstream open reading frame (uORF) located from -521 to -432 in human. The uORF is very different from the main p27Kip1 ORF beginning at +1 . The sequence of the uORF resembles that of mini-mRNA embedded in the unusually long 5 '-untranslated region (5'-UTR) of p27Kip1 mRNA. It contains AUG codon on the extreme 5'-side beginning at -521 and UAAAAAAA on the extreme 3 '-side beginning at -432 . In between these two sequences, it contains codons for 29 amino acid residues.

c) The third element is the internal ribosome entry site (IRES). It is placed centered around the polypyrimidine tract (from -66 to -41 in human). This is the site where $40 \mathrm{~S}$ ribosome attaches and begins scanning towards the 5'-AUG of the main p27Kip1 ORF when m7G-5'-cap at the -575 is compromised.

Human p27 5'-UTR (-575 - -1): An Overview

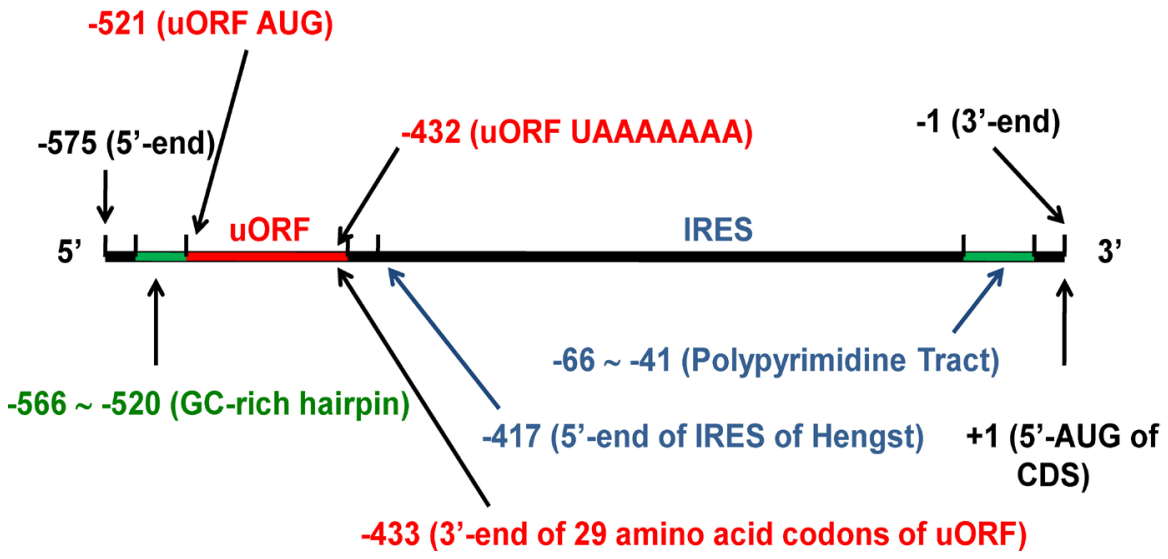

Figure 2. Primary structure of 5'-untranslated region of p27Kip1 mRNA. 


\section{Effect of Anti-Cancer Agents on the Secondary Structure of 5'-Untranslated Region of p27Kip1 mRNA}

\subsection{Effect of Anti-Cancer Agents on the Expression of p27Kip1 Protein (Figure 3)}

The main objective of this section is to describe how anti-cancer agents increase the translation initiation of $\mathrm{p} 27 \mathrm{Kip} 1$ protein by forming a particular secondary structure of the 5'-untranslated region of p27Kip1 mRNA. This is described in the section immediately following this section.

Here in the present section, several relevant scientific findings will be summarized regarding the effect of how anti-cancer agents increase the translation initiation of $\mathrm{p} 27 \mathrm{Kip} 1$ protein.

1) Numerous nutritional and chemopreventive anti-cancer agents increase the expression of p27Kip1 protein in several established cell lines in vitro [1].

2) Anti-cancer agents specifically Increase the expression of p27Kip1 protein without directly affecting the expression of any other cell cycle regulatory proteins including p21Cip1/Waf1. This observation could not be made using western immunoblot analysis of various cell cycle regulatory proteins. It could only be made by using luciferase reporter plasmid constructs of proximal 5'-region of the DNA of various cell cycle regulatory proteins [1] [2].

3) Anti-cancer agents increase the expression of p27Kip1 protein primarily at the level of translation, not at the level of transcription [1] [2].

4) Anti-cancer agents increase the relative luciferase activity of the luciferase reporter plasmid constructs of the 5'-untranslated region of p27Kip1 DNA. The 5'-untranslated region of p27Kip1 DNA does not contain any cryptic transcription factors. This has been established primarily by adding actinomycin $\mathrm{D}$, a transcriptional inhibitor, in the luciferase assay of the 5 -untranslated region of p27Kip1 DNA [2].

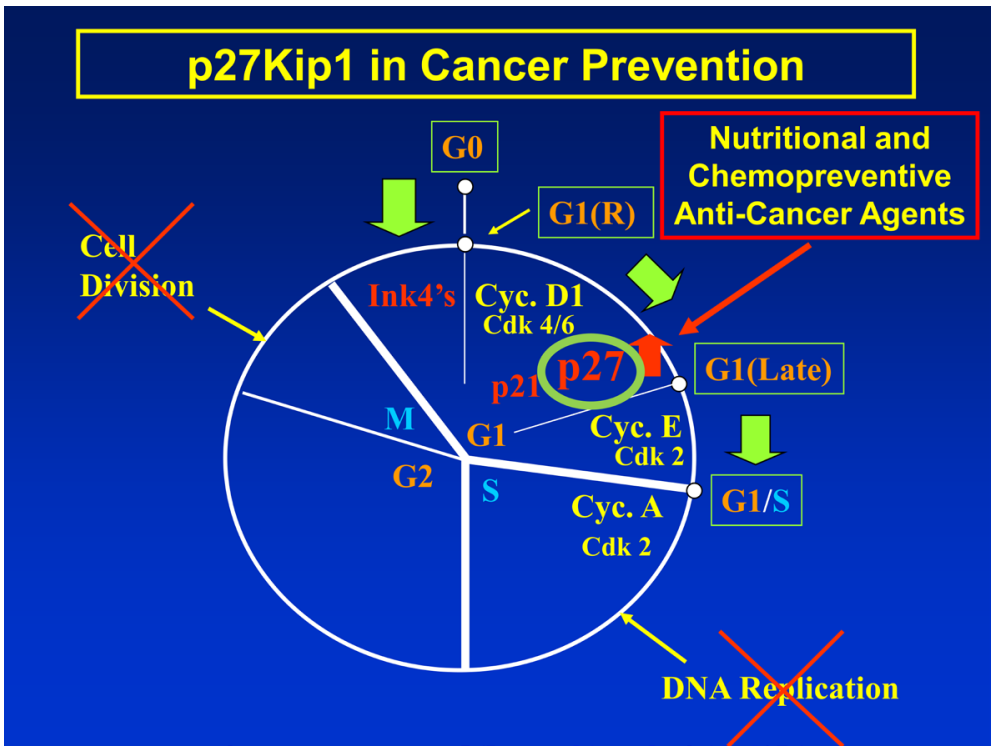

Figure 3. Effect of anti-cancer agents on the expression of p27Kip1 protein. 


\subsection{Effect of Anti-Cancer Agents on the Secondary Structure of 5-Untranslated Region of p27Kip1 mRNA (Figure 4)}

At this point, Dr. Albert Einstein's "visualized thought experiments (German: Gedanken experiment)" will be used as a fundamental tool for understanding how anti-cancer agents modify the primary structure of the 5 '-untranslated region of p27Kip1 mRNA into secondary structure, thereby increasing the translation initiation of p27Kip1 protein.

The results of the visualized thought experiments suggest that:

1) Step 1-Anti-cancer agents compromise the m7G-5'-cap of the 5'-untranslated region (5'-UTR) of p27Kip1 mRNA at -575 . Every pathway leading from anti-cancer agents to 5 '-untranslated region of p27Kip1 mRNA involves a metabolic step that compromises the m7G-5'-cap [1] [2] [3] [4] [5].

2) Step 2 -Step 1 makes the $40 \mathrm{~S}$ ribosome bypass the upstream open reading frame (uORF).

3) Step 3-In the absence of uORF, $40 \mathrm{~S}$ ribosome binds to the downstream internal ribosome entry site (IRES).

4) Step 4-Subsequently, $40 \mathrm{~S}$ ribosome scans the remainder of the 5'-untranslated region (5'-UTR) of p27Kip1 mRNA until it encounters the UAG codon of the main p27Kip1 ORF at the position +1 , thereby up-regulating the translation initiation of p27Kip1 protein.

\section{Effect of Pro-Cancer Agents on the Secondary Structure of 5'-Untranslated Region of p27Kip1 mRNA}

\subsection{Effect of Pro-Cancer Agents on the Expression of p27Kip1 Protein (Figure 5)}

The main objective of this section is to describe how pro-cancer agents (not

\section{Cap-Independent Translation Initiation of p27Kip1 mRNA}

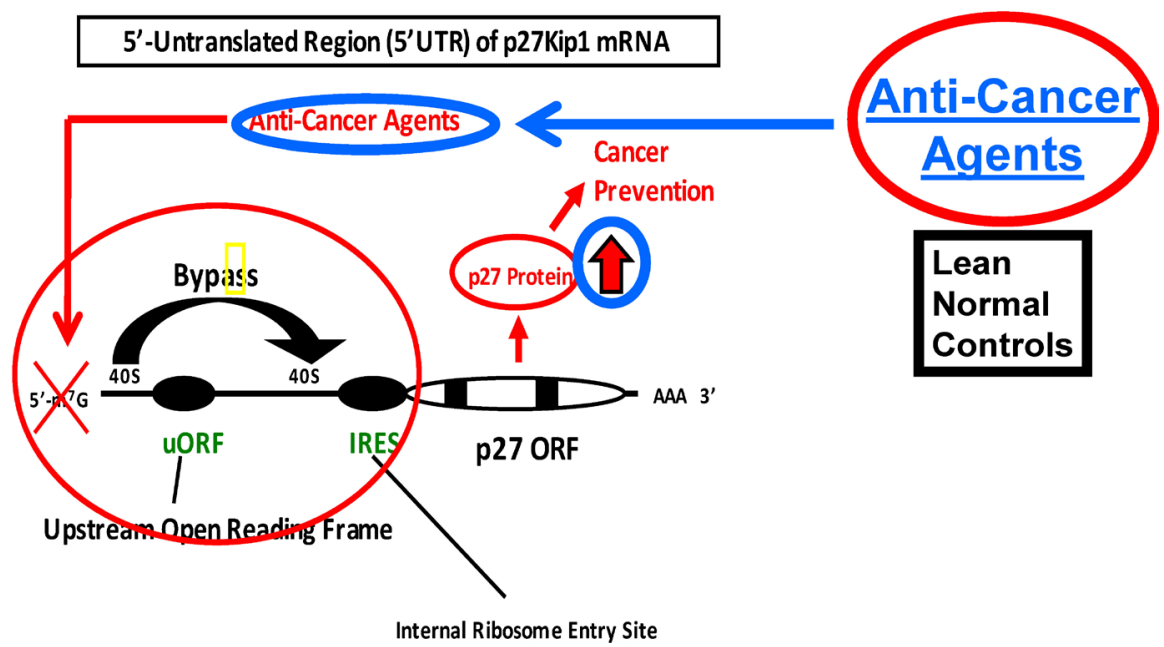

Figure 4. Effect of anti-cancer agents on the secondary structure of 5-untranslated region of p27Kip1 mRNA. 


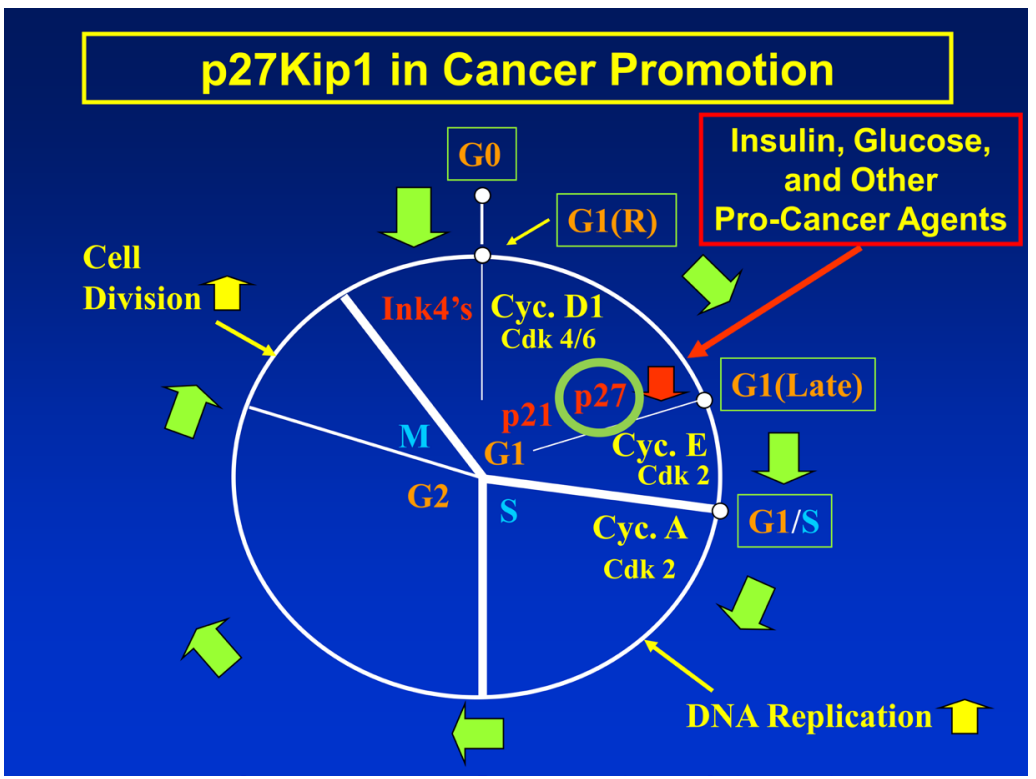

Figure 5. Effect of pro-cancer agents on the expression of p27Kip1 protein.

anti-cancer agents) decrease (not increase) the translation initiation of p27Kip1 protein by forming an alternative secondary structure of the 5 '-untranslated region (5'-UTR) of p27Kip1 mRNA. The most likely mechanism is presented in the section immediately following this section.

Here in the present section, several relevant scientific findings will be summarized regarding the effect of how pro-cancer agents decrease the translation initiation of $\mathrm{p} 27 \mathrm{Kip} 1$ protein.

1) Almost all scientific findings described above for the anti-cancer agents are applicable to the pro-cancer agents with the following one major technical difference [1] [2] [3] [4].

2) The technical difference between the pro-cancer agents and anti-cancer agents derives primarily from the different chemical structures and origins of these two types of agents. More specifically speaking, all of the anti-cancer agents identified so far have turned out to be foreign to our body, whereas, the pro-cancer agents identified so far have turned out to be native to our body. Thus, the fundamental technical difficulties of identifying pro-cancer agents derive from the fact that they are native to our body. For example, glucose in the established cell lines in vitro could be identified as a pro-cancer agent only by using luciferase reporter plasmid constructs of the 5'-untranslated region (5-UTR) of p27Kip1 mRNA [1] [2]. Whereas, glucose could be readily identified as a pro-cancer agent in the peripheral blood mononuclear cells (PBMC) obtained from obese, type 2 diabetic patients by performing western immunoblot analysis of the p27Kip1 protein in these cells [5].

\subsection{Effect of Pro-Cancer Agents on the Secondary Structure of 5-Untranslated Region of p27Kip1 mRNA (Figure 6)}

The visualized thought experiments described above will be used again as a 


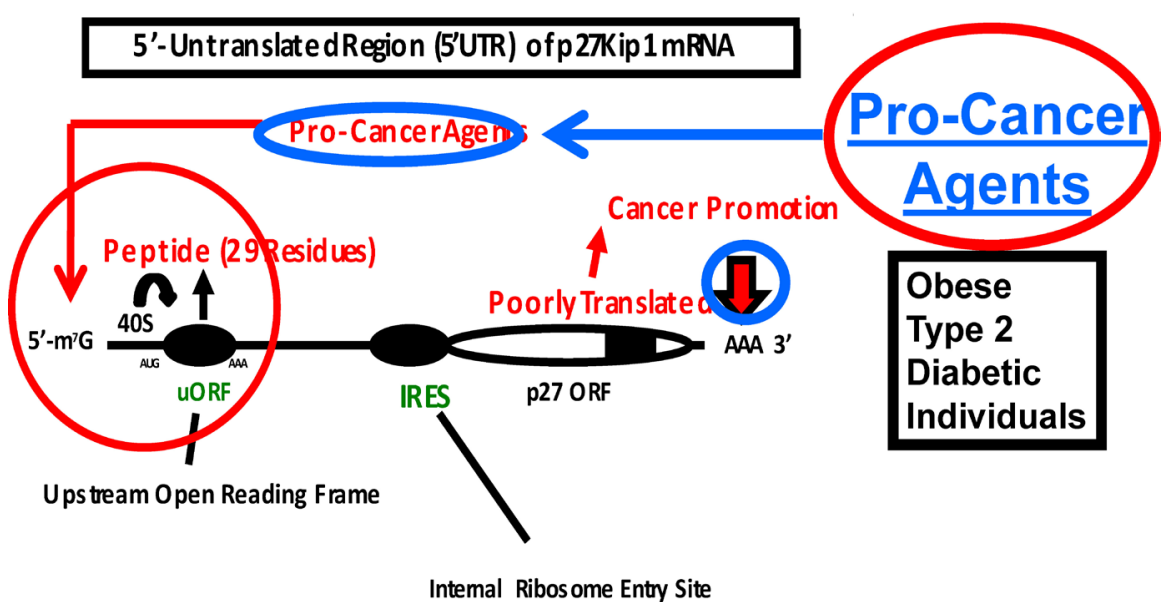

Figure 6. Effect of pro-cancer agents on the secondary structure of 5-untranslated region of p27Kip1 mRNA.

fundamental tool for understanding how pro-cancer agents modify the primary structure of the 5'-untranslated region of p27Kip1 mRNA into an alternative secondary structure, thereby decreasing the translation initiation of p27Kip 1 protein.

The results of these experiments are presented below:

1) Step 1-Pro-cancer agents do not compromise the m7G-5'-cap of the 5'-untranslated region (5'-UTR) of p27Kip1 mRNA at -575.

2) Step 2-Step 1 makes the $40 \mathrm{~S}$ ribosome scan the 5 '-untranslated region (5'-UTR) of p27Kip1 mRNA until it encounters the UAG codon of the intact upstream open reading frame (uORF) (not the UAG codon of the main p27Kip1 ORF at the position +1 ).

3) Step 3-Once 40S ribosome encounters the UAG codon of the intact uORF, $60 \mathrm{~S}$ ribosome is recruited to the UAG codon and starts translating 29 amino acid codons into 29 amino acid residues.

4) Step 4-After the Step 3, 40S-60S ribosome complex on the uORF encounters the terminating codon and poly-A at the 3 '-end of uORF. This results in both $40 \mathrm{~S}$ and $60 \mathrm{~S}$ ribosomes detach from the uORF and separated.

5) Step 5-As a result of the $40 \mathrm{~S}$ ribosome detaching from the uORF, it could never scan and reach the UAG codon of the main p27Kip1 ORF at the position +1 . This down-regulates the translation initiation of p27Kip1 protein.

\section{Conclusions}

Unlike all other cell cycle regulatory proteins, p27Kip1 protein is uniquely suited to handle either anti- or pro-cancer agents for the following two reasons. 1) Either anti- or pro-cancer agents specifically increase or decrease the expression of only p27Kip1protein, no other cell cycle regulatory proteins. 2) The mRNA of p27Kip1 is the only cell cycle regulatory protein that has a long, unusual 5 '-untranslated region that could handle either stimulation or inhibition of the translation initiation of p27Kip1 protein, no mRNAs of all other cell cycle regu- 
latory proteins.

The structure of this long, unusual 5'-untranslated region of p27Kip1 mRNA is unfortunately rather complex. So, we do not know for sure how this 5 '-untranslated region of p27Kip1 mRNA could stimulate or inhibit the translation initiation of $\mathrm{p} 27 \mathrm{Kip} 1$ protein.

The short concept proposal presented in this manuscript is intended to provide the reader with fairly useful and clear guidance as to how the actual experiments should be conducted.

\section{Conflicts of Interest}

The authors declare no conflicts of interest regarding the publication of this paper.

\section{References}

[1] Eto (2006) Nutritional and Chemopreventive Anti-Cancer Agents Up-Regulate Expression of p27Kip1, a Cyclin-Dependent Kinase Inhibitor, in Mouse JB6 Epidermal and Human MCF7, MDA-MB-321 and AU565 Breast Cancer Cells. Cancer Cell International, 6, 2. https://doi.org/10.1186/1475-2867-6-2

[2] Eto (2010) Upstream Molecular Signaling Pathways of p27(Kip1) Expression: EFFECTS of 4-Hydroxytamoxifen, Dexamethasone, and Retinoic Acids. Cancer Cell International, 10, 3. https://doi.org/10.1186/1475-2867-10-3

[3] Eto (2011) Upstream Molecular Signaling Pathways of p27 (Kip1) Expression in Human Breast Cancer Cells in Vitro: Differential Effects of 4-Hydroxytamoxifen and Deficiency of Either D-(+)-Glucose or L-Leucine. Cancer Cell International, 11, 31. https://doi.org/10.1186/1475-2867-11-31

[4] Eto (2013) Expression of p27Kip1, a Cell Cycle Repressor Protein, Is Inversely Associated with Potential Carcinogenic Risk in the genetic Rodent Models of Obesity and Long-Lived Ames Dwarf Mice. Metabolism Clinic and Experimental, 62, 873-887.https://doi.org/10.1016/j.metabol.2013.01.001

[5] Eto (2014) Expression of p27(Kip1), a Cyclin-Dependent Kinase Inhibitor, in Human Peripheral Blood Mononuclear Cells Is Inversely Associated with Potential Carcinogenic Risk in Obese Type 2 Diabetic Individuals Relative to Lean Normal Controls. American Journal of Molecular Biology, 4, 114-128.

https://doi.org/10.4236/ajmb.2014.43013

\section{List of Abbreviations}

5'-cap: five-prime cap;

5'-UTR: 5'-untranslated region;

IRES: internal ribosome entry site;

m7G-5'-cap: 7-methylguanylated five-prime cap;

mRNA: messenger RNA;

ORF: open reading frame;

UAG codon: translation initiation codon;

uORF: upstream open reading frame. 